\title{
Revisión nacional e internacional de las competencias profesionales de los docentes de Educación Física National and international review of Physical Education teachers' professional competences \\ *Inmaculada Rodriguez Gomez, **Sagrario Del Valle, ***Ricardo De la Vega Marcos \\ *Centro Universitario Villanueva (Universidad Complutense de Madrid), **Universidad de Castilla La Mancha (España), ***Universidad
} Autónoma (Madrid)

\begin{abstract}
Resumen. Se presenta un estudio sobre las competencias docentes con el fin de llegar a hacer una aproximación sobre qué competencias debe poner en práctica el profesorado de educación física que permita definir un perfil docente excelente y eficaz. Para conseguir este objetivo, se ha llevado a cabo una revisión de los marcos para la buena enseñanza a nivel nacional e internacional e investigaciones sobre las competencias profesionales de los docentes tanto a nivel general como en concreto de la educación física. Para ello, se decide adoptar el modelo competencial docente más aceptado en la literatura científica que las agrupa en cuatro dimensiones: Conocimiento del contenido de enseñanza, Conocimiento didáctico del contenido, Gestión/ organización de la clase y la capacidad de liderazgo del docente y de relación con otros agentes. Se ha llegado a la conclusión, hasta la fecha, no se halla en la literatura revisada ningún instrumento validado que evalúe la confirmación de las cuatro competencias especificadas. Máxime cuando los cambios tan vertiginosos que sufre la sociedad actual, demanda un modelo de profesor que sepa adaptarse a los cambios poniendo en práctica un liderazgo creativo donde la relación con el estudiante ha de ser de reciprocidad durante todo el proceso enseñanza-aprendizaje.

Palabras clave: competencias, docentes, educación física, reciprocidad y liderazgo creativo.
\end{abstract}

Abstract. A study about teaching competences is presented with the aim to get to an approximation of the competences advocating excellent and effective teaching profile when put into practice by physical education teachers. To achieve this goal, two actions were taken: a review of the frameworks for good teaching at national and international level, and a research on teachers' professional competences both at a general level and in particular in physical education. Based on the most accepted teacher competence model in the scientific literature, four dimensions were identified: Knowledge of teaching content, didactic knowledge of content, Management / organization of the classroom, and teachers' leadership capacity and relationship with other agents. As a conclusion, no validated instrument evaluating the confirmation of the four specified competences was found in the reviewed literature. Our society keeps a frenetic pace nowadays, therefore it demands a model of teacher who can adapt to changes and display creative leadership while building reciprocal relationships with students throughout the teaching-learning process.

Key words: competences, teachers, physical education, reciprocity and creative leadership.

\section{Introducción}

El cambio social de los últimos años, provoca mayores demandas en el profesorado del siglo XXI. Los sistemas educativos deben tratar de garantizar que las personas cuenten con las competencias que se requieren en el mercado laboral (OCDE, 2016). Ante esta afirmación, hay que pensar que los docentes requieren de unas competencias profesionales que les permita enfrentarse al nuevo contexto educativo para que puedan llevar a cabo una educación de calidad, surgiendo la necesidad de investigar cuáles son las características que constituyen un profesor eficaz capaz de hacer frente a las demandas de la sociedad actual.

En esa búsqueda de la calidad educativa, se realiza una revisión de los descriptores que configuran las competencias profesionales docentes desde las entidades y asociaciones que velan por las buenas prácticas en el desempeño docente en España y en diferentes países extranjeros, así como las investigaciones e instrumentos de evaluación utilizados en este ámbito, para descubrir los indicadores que pueden aportar luz sobre los rasgos que definen las competencias profesionales capaces de dibujar y justificar un modelo que nos reporte posibles éxitos en el proceso de enseñanza- aprendizaje.

Delimitar las competencias docentes y el modo de evaluarlo es una cuestión muy compleja por los aspectos personales del docente y el contexto en el que se desarrolla su labor.

Poco a poco se va extendiendo la importancia de la evaluación del profesorado como medio para su desarrollo profesional. Entendemos la evaluación como medio para ayudar al docente a mejorar su desempeño, identificando sus logros y detectando sus problemas, perspectiva que coincidiría con la evaluación formativa para el desarrollo profesional.

Para poder establecer un marco de estudio científico se van a revisar las competencias docentes desde una perspectiva internacional y nacional, y se van a poner de manifiesto los instrumentos de evaluación que hasta la fecha conocemos respecto a esta cuestión, tomando como referencia la didáctica general y en concreto la didáctica de la educación física.

Fecha recepción: 03-06-17. Fecha de aceptación: 18-12-17 Sagrario Del Valle

Sagrariodel.Valle@uclm.es
Conseguir llegar a delimitar un marco de competencias, conlleva un gran esfuerzo y un giro en las políticas educativas a nivel mundial.

Por este motivo, la revisión que a continuación se presenta es por un lado de aquellos países y organismos que están a la brecha en educación y por otro los que sí manifiestan su intención en mejorar y delimitar el perfil docente de sus profesionales.

Como expresa Álvarez (2015, p.10), «fueron precisamente los organismos internacionales quienes alertaron de la necesidad de preocuparnos por la calidad del profesorado para mejorar los sistemas educativos, elevando a prioritario un tema que ya estaba candente en la literatura académica y científica con anterioridad».

¿Y qué se entiende por perfil docente? Galvis, Fernández y Valdivieso (2006, p. 31), el conjunto de competencias necesarias para realizar su actividad profesional, «de acuerdo con criterios valorativos y parámetros de calidad». Es por tanto, concebir los elementos cognitivos, de destrezas y actitudinales que le configuran como profesional. O lo que es lo mismo, un marco de referencia en el que están recogidas sus competencias (Sánchez-Tarazaga, 2016).

\section{Estado actual del tema}

\subsection{Revisión internacional de las competencias profesionales de} los docentes

Las tareas o actividades que realizan los docentes se han de modificar y ampliar, urgiendo la necesidad de revisar su perfil, redefinir su trabajo y formación, así como adaptar su manera de enseñar (FerrándezBerrueco y Sánchez-Tarazaga, 2014; Marcelo, 2002; Martínez, 2016).

¿Cómo decidir la importancia de unas competencias respecto a otras? ¿Es posible consensuar las características clave de dichas competencias? ¿Podemos encontrar marcos de competencias docentes ya definidos en el contexto internacional?

La Comisión Europea (2013), concibe la profesión en cuatro ámbitos aportando unas orientaciones en la construcción de un perfil competencial docente entendiendo las competencias como una integración dinámica de habilidades cognitivas y metacognitivas:

- Aprender a pensar como docentes: implica una revisión crítica de las creencias de uno mismo y el desarrollo del pensamiento pedagógico.

- Aprender a saber cómo docentes: se refiere a diferentes aspectos 
del conocimiento, incluyendo el que se genera por las propias prácticas.

- Aprender a sentir como docentes: vinculado directamente con la identidad profesional, incluye aspectos emocionales e intelectuales como las actitudes, expectativas, liderazgo y valores.

- Aprender a actuar como docentes: supone integrar pensamientos, conocimientos y actitudes en la práctica y en contextos diferentes.

No obstante, Sánchez-Tarazaga (2016, p.58), pone de relieve la necesidad de concebir el perfil docente más allá de experto del conocimiento como puede ser la colaboración con la comunidad educativa (profesores, familias y otros agentes), la participación en el centro o la gestión de la diversidad, entre otras.

Bajo esta idea, se entiende que en futuros trabajos de investigación, resulta de gran interés ampliar este paisaje con otros modelos educativos vigentes, que a buen seguro puedan complementar este análisis.

En Europa y en concreto en Holanda, con el consenso de las asociaciones profesionales de docentes, se establecen sieteámbitos de competencias y sus descriptores que debe mostrar el profesorado como referente de un adecuado sustrato para una buena enseñanza (SBL, 2003).

En Eslovenia, el estudio que realizan Bourgonje y Tromp (2011, pp. 115-116), desarrollan un modelo donde clasifican las competencias docentes en 5 dimensiones (Ver Tabla 1).

En América Latina, el modelo de los estándares de desempeño profesional docente del Ministerio de Educación de Ecuador (2011), está compuesto por cuatro dimensiones quellevan a mejorar el aprendizaje de los estudiantes. El marco para la buena enseñanza del Ministerio de Educación de Chile (2003) estructura cuatro dominios de competencias. El perfil del docente de Educación Media Superior de la Reforma integral de México (2008), está formado por ocho competencias. En Guatemala el proyecto USAID (2011), marca una clasificación con cuatro dimensiones competenciales. El Marco de Buen desempeño docente de Perú (2012), marca cuatro dimensiones y en la República Dominicana en su Modelo de evaluación del desempeño docente basado en competencias (2012), marcan dos grandes dimensiones (Ver Tabla 2).

En EEUU, el Marco para la Enseñanza de

Fuente: Elaboración propia

Tabla 2

Dimensiones competencias docentes América Latina

\begin{tabular}{ll}
\hline PAÍS & a) Desarrollo curricular: compuesto por tres descripciones generales de desempeño docente que son necesarios para poder planificar y enseñar: (1) dominar el área del
\end{tabular} saber que enseña; (2) comprender y utilizar las principales teorías e investigaciones relacionadas con la enseñanza y su aprendizaje; (3) comprender, implementar y gestionar el currículo nacional.

b) Desarrollo profesional: compuesto por cuatro descripciones generales de desempeño docente que son necesarios para la enseñanza: (1) planificar el proceso de enseñanza- aprendizaje; (2) crear un clima de aula adecuado para la enseñanza y el aprendizaje; (3) interactuar con los alumnos en el proceso de enseñanza-aprendizaje; (4) evaluar, retroalimentar, informar e informarse de los procesos de aprendizaje de los estudiantes.

c) Gestión del aprendizaje: compuesto por tres descripciones generales de desempeño docente que son necesarios para el desarrollo profesional: (1) mantenerse actualizado respecto a los avances e investigaciones en la enseñanza de su área del saber; (2) colaborar con otros miembros de la comunidad educativa; (3) reflexionar acerca de su labor, sobre el impacto de la misma en el aprendizaje de sus estudiantes.

d) Compromiso ético: compuesto por cuatro descripciones generales de desempeño docente que son necesarios para su desarrollo profesional: (1) tener altas expectativas respecto al aprendizaje de todos los estudiantes; (2) comprometerse con la formación de sus estudiantes como seres humanos y ciudadanos en el marco del

\begin{tabular}{l} 
"buen vivir"; (3) enseñar con valores garantizando el ejercicio permanente de los derechos humanos; (4) comprometerse con el desarrollo de la comunidad más cercana. \\
\hline CHILE preparación de la enseñanza; \\
b) creación de ambientes propicios para el aprendizaje; \\
c) enseñanza para el aprendizaje de todos los alumnos; \\
d) responsabilidad profesional. \\
En cada uno de los dominios se establecen criterios que identifican los conocimientos, habilidades y actitudes, así como las funciones que debe saber movilizar el
\end{tabular} d) responsabilidad profesional.

En cada uno de los dominios se establecen criterios que identifican los conocimientos, habilidades y actitudes, así como las funciones que debe saber movilizar el docente para el logro de la buena enseñanza y el propio desarrollo profesional.

MÉXICO $\quad$ a) organiza su formación continua a lo largo de su trayectoria profesional;

c) planifica los procesos de enseñanza y de aprendizaje atendiendo al enfoque por competencias, y los ubica en contextos disciplinares, curriculares y sociales amplios;

c) lleva a la práctica procesos de enseñanza y de aprendizaje de manera efectiva, creativa e innovadora a su contexto institucional;

e) evalúa los procesos de enseñanza y de aprendizaje con un enfoque formativo;

f) construye ambientes para el aprendizaje autónomo y colaborativo;

g) contribuye a la generación de un ambiente que facilite el desarrollo sano e integral de los estudiantes;

h) participa en los proyectos de mejora continua de su escuela y apoya la gestión institucional.

GUATEMALA $\quad$ h) participa en los proyectos de mejora continua de su escuela y apoya la gestion institucional. a) Competencia interpersonal: el docente demuestra estar comprometido con la equidad, la igualdad y la ética, que correspon

b) Competencia didáctica: comprometido con el aprendizaje y el desarrollo integral de los estudiantes, tomando en cunas. b) Competencia didáctica: comprometido con el aprendizaje y el desarrollo integral de los estudiantes, tomando en cuenta su identidad cultural, étnica y lingüística, se refiere a la adecuada comunicación con sus estudiantes y

c) Competencia disciplinar: copia pa disciplinas que enseña y el arte de enseña para el desarrollo y c) Competencia disciplinar: comprometido con su continua actualización en los conocimientos de las disciplinas que enseña, y el arte de enseñar para el desarrollo y formación integral de sus estudiantes; alude al buen dominio que el docente debe tener de los contenidos de las áreas curriculares que imparte y a la forma como el
docente guía el aprendizaje de sus alumnos, según el contexto sociocultural. docente guía el aprendizaje de sus alumnos, según el contexto sociocultural.

d) Competencia profesional: comprometido con la calidad en su desempeño docente y crecimiento profesional, se relaciona con el ejercicio docente y profesional tanto

\begin{tabular}{ll} 
en las actividades escolares como con otros miembros de la comunidad educativa. \\
\hline PERÚ & a) Preparación para el aprendizaje de los estudiantes: comprende las características de los estudiantes y conoce los contenidos disciplinares.
\end{tabular}

b) Enseñanza para el aprendizaje de los estudiantes: crea un clima propicio para el aprendizaje, conduce el proceso de enseñanza con dominio de los contenidos disciplinares, con el fin del aprendizaje reflexivo y crítico, evalúa el aprendizaje de los alumnos, teniendo en cuenta las diferencias individuales.

c) Participación en la gestión articulada de la escuela a la comunidad: participa activamente en la gestión de la escuela mejorando el proyecto educativo generando aprendizajes de calidad, establece relaciones de respeto y colaboración con las familias, comunidad y otras instituciones del estado.

d) Desarrollo de la profesionalidad y la identidad docente: reflexiona sobre su práctica y experiencia y ejerce su profesión éticamente.

REPÚBLICA a) Competencias funcionales docentes: conocimiento de la materia que enseña, conocimiento de las áreas transversales del currículo, conocimiento de las Tics y la

DOMINICANA incorporación a su práctica pedagógica, habilidades y capacidades didácticas y pedagógicas para implementar situaciones de aprendizaje significativos en el aula; habilidades del profesor para promover un clima de aula positivo y de aprendizaje significativo; habilidades de programación y desarrollo (planificación del curso, selección y creación de materiales; utilización de los recursos disponibles), capacidades y habilidades evaluativas (evaluación del proceso de enseñanza-aprendizaje de los alumnos y de su progreso); evaluación de los materiales y del currículo.

b) Competencias de profesionalidad docente: capacidad de reflexionar sobre la propia práctica pedagógica, habilidades para el ejercicio de liderazgo transformador dentro de la comunidad educativa, habilidades de ética profesional del docente, conocimiento acerca de la profesión docente (normativas y reglamentaciones del sistema educativo, derechos y deberes como profesional docente, conocimiento de la escuela y su entorno), habilidades y capacidades relacionales (establecimiento de 


\begin{tabular}{|c|c|}
\hline OCEANÍA & DIMENSIONES \\
\hline & $\begin{array}{l}\text { 1. Utilización de estrategias y técnicas de enseñanza innovadoras para resolver con } \\
\text { más eficacia las necesidades de aprendizaje de los estudiantes individuales y de los } \\
\text { grupos de estudiantes; } \\
\text { 2. Empleo de prácticas ejemplares en la evaluación del estudiantes y en la } \\
\text { información de los procesos; } \\
\text { 3. Implicación del profesor en una variedad de actividades de autodesarrollo, } \\
\text { incluyendo un alto nivel sistemático de reflexión crítica sobre su práctica de } \\
\text { enseñanza, sosteniendo así un alto nivel de crecimiento profesional; } \\
\text { 4. Existe una mejora del conocimiento y de las habilidades profesionales de otros } \\
\text { profesores a través del empleo de estrategias eficaces de desarrollo; } \\
\text { se da un alto nivel de liderazgo en la comunidad escolar, asumiendo un papel clave } \\
\text { en procesos de desarrollo de la escuela, incluyendo la planificación y la gestión del } \\
\text { currículo y la formulación de la política escolar. }\end{array}$ \\
\hline
\end{tabular}

Tabla 5

Dimensiones competencias docentes Uganda, Mali e India

Tabla 3 Dimensiones competencias do $\frac{\text { PAÍS }}{\text { UGAND }}$

1.Planificación y pr pedagogía, demostra pedagógico, demostr coherente, diseño de 2.El ambiente del establecimiento de procedimientos de a espacio físico.

3.Instrucción: comu debate, compromiso $\quad \begin{array}{ll}\text { anstrucción, demostri } \\ \text { MALI } & \text { 1.Competencias disciplinarias o interdisciplinarias: dominar los contenidos }\end{array}$ 4.Responsabilidades registros rigurosos, 1 OHIO $\quad$ 1.Planificación docer 2.Evaluación del pr objetivo de ajustar s habilidades que pres 3.Usa el conocimient 4.Conoce el contexto 5.Explica el contenid 6.Atiende a la divers 7.Utiliza recursos val INDIA 8.Crea un buen ambi 9.Evalúa el aprendiz 10.Tiene responsabil $\frac{\text { autoevalúa su práctic }}{\text { Fuente: Elaboración propia. }}$
1.Observar e implicar a los niños, comunicarse y relacionarse con ellos. 2.Comprenderse a sí mismos y a los demás, sus creencias, asunciones, emociones y aspiraciones; desarrollar la capacidad de autoanálisis, autoevaluación, adaptabilidad, flexibilidad, creatividad e innovación.

3.Desarrollar unos hábitos y una capacidad de aprendizaje autónomo, tener tiempo para pensar, reflexionar, asimilar y articular nuevas ideas; ser críticos consigo mismos y colaborar en grupo.

4.Implicarse con el contenido del tema, analizar los conocimientos de la disciplina y las realidades sociales, relacionar la asignatura con el entorno social de los alumnos y desarrollar el pensamiento crítico.

1.Competencias disciplinarias o interdisciplinarias: dominar los contenidos académicos, los métodos relacionados con cada disciplina y los vínculos que puedan existir entre las disciplinas.

2.Competencias pedagógicas: intervenir eficazmente y a favor del aprendizaje de los alumnos. Se trata de competencias pedagógicas en psicología del aprendizaje, didáctica y tecnologías de la educación, comunicación, evaluación y gestión del aula

Competencias profesionales: permiten al docente integrarse bien en la profesión y actuar en conformidad con la ética profesional.

4.Competencias socio-culturales o socio-relacionales: permiten al docente promove los valores sociales de respeto y dignidad de la persona, y desempeñar el papel que se espera de él en un entorno más amplio

Cuidar a los niños: comprenderlos en los contextos sociales, culturales y políticos, desarrollar sensibilidad hacia sus necesidades y problemas y tratarlos en igualdad de condiciones.

2.No percibir a los niños como receptores pasivos de conocimientos: aumentar su propensión natural a construir significados, desalentar el aprendizaje de memori ndizaje sea una actividad divertida, participativa y significativa.

3.Analizar de forma crítica los currículos y los libros de texto, contextualizar el currículo para adaptarse a las necesidades locales.

4.No tratar el conocimiento como algo "dado", integrado en el currículo y aceptado sin cuestionar.

5.Organizar experiencias de aprendizaje participativas, centradas en el alumno y basadas en actividades como jugar, hacer proyectos, debatir, dialogar, observar, visitar y aprender a reflexionar sobre su propia práctica.

6.Integrar el aprendizaje académico en las realidades sociales y personales, 6.Integrar el aprendizaje académico en

7.Promover valores de paz, una forma de vida democrática, igualdad, justicia, 7.Promover valores de paz, una forma de vida democrática, igualdad, justicia,
libertad, fraternidad, laicismo y entusiasmo por la reconstrucción social. Fuente: Elaboración propia

Danielson (2011, p. 16) aporta cuatro dominios y en concreto, los Estándares de desempeño docente del Departamento deEducación del Estado de Ohio (2015) aportan las siguientes dimensiones competenciales (VerTabla 3).

En Oceanía, desde el punto de vista del grado elevado del desempe- ño docente, el Departamento de Educación de Australia Oeste en 2009, comienza a poner en marcha el programa Level 3 Classroom Teacher como una propuesta que identifica a docentes que llevan a cabo una buena tarea profesional. Dicho programa se estructura en torno a ámbitos de competencias, siendo en este caso cinco los grupos establecidos que se conforman con cuatro o cinco descriptores. Elementos que permiten llevar a cabo una evaluación oficial o una autoevaluación (Hernández y Velázquez, 2010) (Ver Tabla 4).

Además, países como Uganda, el Perfil de competencias de un maestro de primaria de Mali y el Marco curricular Nacional de la India, describen lo que deberían saber y hacer los profesionales (Bourgonje y Tromp, 2011, p. 84-124) (Ver Tabla 5).

\subsection{Revisión nacional de las competencias profesionales de los} docentes

Si hacemos ahora una revisión de las competencias profesionales de los docentes teniendo en cuenta las diferentes comunidades autónomas en España, hallamos el modelo de competencias profesionales del profesorado de la Red de Formación del profesorado de Castilla y León (2010), donde expone diez competencias profesionales del profesorado clasificadas dentro del modelo europeo (Comitémixto OIT/UNESCO, Eurydice, OCDE, Comisión de las Comunidades Europeas), identificando cinco ámbitos. El Modelo competencial de la Red de Formación del Profesorado de la Xunta de Galicia (2014), define siete competencias. El Departamento de Educación, Cultura y Deporte del Gobierno de Aragón (2013), en la Guía para la evaluación de la función docente donde se explicitan las dimensiones, subdimensiones e indicadores para evaluar al profesorado, identificando dos categorías clave. En la Junta de Andalucía desde la Consejería de Educación (2011), establece los estándares de la práctica profesional docente según la Agencia andaluza de evaluación educativa. La Consejería de Educación de la Generalitat Valenciana (2010), propone unos descriptores para la evaluación del profesorado en los niveles no universitarios y la valoración práctica docenteque desde la inspección educativa de la Consejería de Educación de la Comunidad de Madrid (2001), lleva a cabo para evaluar al profesorado, lo hace atendiendo a dos dimensiones. En el informe del Consejo Escolar de Castilla-La Mancha sobre «El educador en la sociedad del siglo XXI» (2007), se señalan algunos rasgos que definen el modelo de profesor que se está demandando la sociedad (Ver Tabla 6).

Además de las competencias docentes que marcan diferentes $\mathrm{CO}$ munidades autónomas, existen investigaciones en el ámbito de la educación y en concreto en la educación física, que nos ayudan a encontrar la manera de organizar las competencias para encontrar un modelo de perfil profesional docente.

\subsection{Instrumentos de evaluación de las competencias profesiona-} les de los docentes y los docentes de educación física

Veamos los estudios en el ámbito de la didáctica general:

Bridges (1986), agrupa las competencias docentes en cinco grandes categorías. Jofré y Gairín (2010), citado en Gairín (2011, pp. 93-108), analizan las competencias deseables para un docente. Además se presenta el resultado de un trabajo en equipo por grandes redes profesionales como el Informe Tuning de la UNESCO sintetizando las competencias profesionales docentes. Díaz et al. (2010), presentan un Modelo de autoevaluación de las competencias docentes para maestros (Ver Tabla 7).

Si nos ceñimos ahora en el ámbito de la educación física hallamos las siguientes investigaciones, basándonos en la didáctica específica como la que aporta Hernández y Velázquez (2010, pp. 69-94), corroborado por investigaciones de autores como Del Valle, De la Vega y Rodríguez (2015) y Ferrández-Berrueco y Sánchez-Tarazaga (2014). Lavega (2008, pp. 123-131), muestra las competencias que debe poseer un profesor de educación física en secundaria. Carreiro da Costa (2006, p. 39), haciendo un análisis del Proyecto AEHESIS (Aligning a European Higher Education Structure In Sport Science), aporta las competencias que un profesor de educación física debe ser capaz de poseer y cumplir (Ver Tabla 8) 
Tabla 6

Competencias docentes por Comunidades Autónomas

COMUNIDAD

AUTÓNOMA

CASTILLAY a)Saber o competencia científica: se relaciona con el conocimiento y la gestión del mismo, tanto en el área de educación como en las áreas, las materias y los módulos curriculares.

LEÓN b) Saber ser o competencia intra e interpersonal: se refiere a la propia forma de ser de la persona y a la forma de bien tratar a los demás, a través de habilidades personales, de la acción tutorial, la orientación y la gestión y la promoción de valores.

c)Saber hacer qué o competencia didáctica: se centra en enseñar, prestando atención al proceso de enseñanza aprendizaje y a la gestión del mismo. Esta competencia se operativiza en las programaciones, didácticas específicas de áreas, materias y módulos, atención a la diversidad, gestión de aula, recursos y materiales didácticos y evaluación de los alumnos; competencia organizativa y de gestión que alude a la organización en el trabajo y se vincula con la normativa, la planificación, la coordinación y la gestión de calidad en el centro; competencia en gestión de la convivencia dirigida hacia la asertividad propia, el convivir con los demás y la gestión de la convivencia, a través de la promoción, la mediación y el control de la misma como aspectos fundamentales.

d)Saber hacer cómo o competencia en el trabajo en equipo: vinculada con el desarrollo de trabajos colaborativos con un objetivo común; competencia en la innovación y la mejora que tiene que ver con el desarrollo de procesos de afrontamiento del cambio, su investigación y experimentación, así como el diagnóstico y la evaluación para implementar las propuestas de mejora innovadoras planteadas; competencia comunicativa y lingüística que es aquélla que versa sobre el intercambio de conocimientos, ideas, pensamientos, emociones y sentimientos; comprende la gestión de la información y la transparencia, así como la expresión y la comunicación, tanto en la propia lengua como en lenguas extranjeras; Competencia digital (TIC): se refiere al mundo digital y las tecnologías de la información y la comunicación. Su ámbito se encuentra en el conocimiento de las tecnologías, el uso didáctico de las mismas y la gestió de equipos y redes para el desarrollo profesional, entre otros.

e)Saber estar o competencia social-relacional: centrada en las relaciones sociales entre personas y la participación en la comunidad, a través de la gestión correspondiente.

GALICIA a) El educador como guía en el proceso de aprendizaje y desarrollo del alumno: se refiere al uso consciente por parte del profesor de sus capacidades, habilidades, conocimientos y herramientas para generar y motivar procesos de aprendizaje en el alumnado.

b) El educador miembro de una organización: desempeña las funciones relacionadas con la organización y gestión del centro, aplicación de planes y programas y consecución de objetivos.

c) El educador interlocutor y referente en la comunidad educativa: promueve un ambiente propicio y educativo en la escuela.

d) Investigador e innovador: aplica nuevas ideas, propuestas y prácticas educativas para mejorar las competencias de los alumnos y el centro.

e) Especialista en su materia: tiene capacidades cognitivas para la adquisición, empleo y gestión del conocimiento referido a su área específica y conocimiento pedagógico.

d) Comunicador en lenguas maternas y extranjeras: utiliza la lengua como instrumento oral, escrita, representación e interpretación de la realidad.

\begin{tabular}{ll} 
& e) Es competente en tecnologías de la información y la comunicación: usa las TIC para evaluar, almacenar, producir e intercambiar información y comunicarse a través de las redes. \\
\hline ARAGÓN & a)El proceso de enseñanza aprendizaje: planificación del proceso de enseñanza aprendizaje, desarrollo de la actividad docente, evaluación del proceso de enseñanza aprendizaje.
\end{tabular} b)La dedicación al centro: participación en los órganos colegiados y de coordinación docente, así como la participación en actividades del centro, orientación y tutoría, formación y participación en proyectos de innovación e investigación educativa y el cumplimiento de funciones.

a)Habilidades sociales: liderazgo, comunicación, relación y trabajo en equipo.

b) Gestión del trabajo: planificación, gestión de recursos y evaluación.

c) Desarrollo profesional: orientación a la calidad, aprendizaje a lo largo de la vida. d)Docencia: gestión del currículo, gestión de ambientes de aprendizaje, inclusión educativa, acción tutorial y orientación escolar.

位.

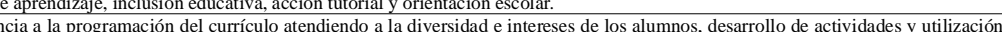

VALENCIANA de diferentes instrumentos y procedimientos de evaluación. Utiliza metodologías innovadoras en el proceso de enseñanza.

b)Actuación y desarrollo profesional: hace referencia a la participación y al cumplimiento de la normativa y la aplicación correcta, participa en equipos de trabajo, en actividades de formación de carácter reglado dirigidas a la actualización de los conocimientos científico-técnicos, didácticos y/o psicopedagógicos, realiza trabajos de investigación e innovación educativa

c)Relación interpersonal: se refiere al conocimiento y comunicación con el entorno de forma adecuada alumno-familia y previene e interviene en la resolución de conflictos facilitando la mejora del clima y la convivencia del centro y el aula.

MADRID a) Dedicación al centro: donde hace referencia a la participación en los órganos colegiados, tener iniciativas para mejorar la práctica docente y el trabajo en equipo, colaborar y poner en marcha actividades extraescolares y complementarias que figuran en la programación general anual del centro, atención o tutoría con alumnos y padres.

b) Actividad docente dentro del aula: valora como el docente prepara la clase y los materiales didácticos en función de la programación, utilización de una metodología de enseñanza adecuada para promover el aprendizaje de los contenidos escolares, procedimientos de evaluación de los aprendizajes que lleva a cabo, y en su caso, de la recuperación e información sobre los mismos a los alumnos y familiares, utilización de medidas ordinarias y extraordinarias para atender a la diversidad de capacidades, intereses y motivaciones de los alumnos, organización del trabajo en el aula para favorecer la adecuada marcha de la clase y la participación e implicación del alumnado en su proceso de aprendizaje.

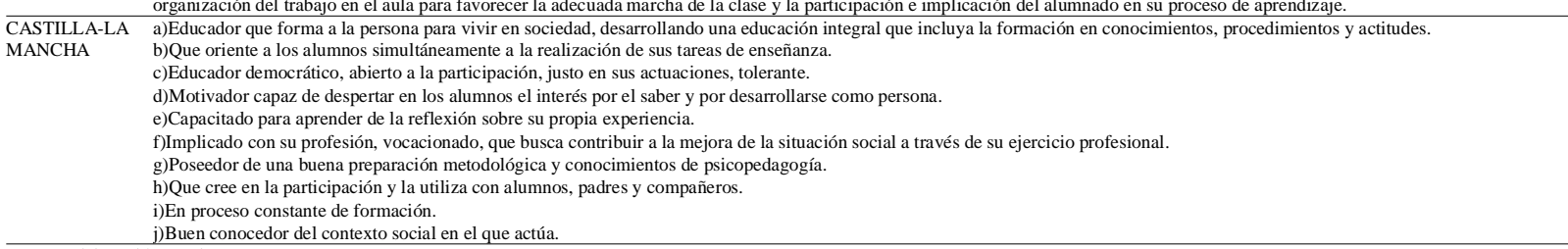

j)Buen conocedor del contexto social en el que actúa. Fuente: Elaboración propia

Tabla 7

Evaluación de las competencias docentes

AuTr

1) Conocimiento de la materia. Referido al dominio que el docente debe poseer sobre su disciplina específica.
2) Capacidad de impartir el conocimiento. En cuanto a su habilidades pedagógicas para llevar a cabo con éxito el proceso enseñanza-aprendizaje.

3) Capacidad de mantener la disciplina en el aula. Saber controlar en todo momento las diferentes situaciones que se presentan en el aula.

4) Capacidad de mantener un clima apropiado en el aula. Poseer estrategias para saber motivar al alumno y al grupo clase hacia el aprendizaje.

5) Capacidad de establecer una relación con los padres y alumnos. Tener habilidades comunicativas.

Jofré y Gairín (2010) 1)Competencias técnicas: saberes relacionados con el quehacer pedagógico y gestión de dispositivos de diferenciación

2)Competencias metodológicas: vinculación del saber con la realidad del alumno e implementación de un proceso reflexivo en la acción educativa.

3)Competencias sociales: disposición para comprender y trabajar junto a otros y opción por un proceso orientado hacia el aprendizaje de los alumnos.

4)Competencias personales: cumplir con la ética de la profesión docente y la responsabilidad en el ejercicio de la profesión.

Díaz et al. (2010) 1)Programación de la enseñanza: planificación, programación larga y corta y contextualización.

2)Metodología y aprovechamiento de recursos: coherencia entre la metodología desarrollada en el aula y la expuesta en la programación, motivación para el aprendizaje,

organización del proceso de enseñanza-aprendizaje, actividades desarrolladas y orientadas del trabajo del alumno y utilización de los recursos del medio.

3)Evaluación de los aprendizajes: evaluación inicial, continua y final, coevaluación y autoevaluación, calificación, promoción, información a familias y alumnos.

4)Formación y evaluación de la enseñanza: formación e innovación educativa, evaluación de la práctica docente.

5)Tutoría: actuaciones con el alumno, contenido, relaciones con padres/madres de alumnos, coordinación con el equipo docente.

6)Atención a la diversidad: recuperación, profundización y enriquecimiento y atención a la diversidad.

7)Clima de aula: distribución del mobiliario y del material en el aula, interacción profesor-alumnos, trabajo en equipo de profesores y la resolución de conflictos en el aula.

Fuente: Elaboración propia.

\section{Discusión}

El presente estudio, ha intentado encontrar un consenso para poder delimitar las competencias que definan el perfil de profesor de educación física que dé respuesta a las demandas de la sociedad actual, como apunta la OCDE (2016).

Para ello ha buscado los puntos en común que pudieran agrupar las competencias profesionales de los docentes provenientes de los diferentes organismos y entidades internacionales y nacionales, que velan por las buenas prácticas, ya que en la investigación no se llega a acuerdos.

En dicho intento se ha adoptado la agrupación de competencias profesionales que aportan Hernández y Velázquez (2010), modelo experiencial no contrastado $(\alpha=, 83)$, por ser la más pertinente en cuanto a las dimensiones que agrupan las competencias docentes. Al aplicar dicho modelo, se observa que no existeninguna investigación validada al respecto que obtenga resultados significativos que ayuden a encontrar dicho perfil. Revisada la literatura publicada revela que es un modelo que presenta limitaciones ya que aporta sólo niveles de percepción en las variables estudiadas, sin poder esclarecer la existencia de diferencias significativas en los estudios. Publicaciones en dicha línea deberían tener en cuenta lo expuesto.

\section{Conclusión}

El sistema educativo sufre grandes tensiones actualmente que son consecuencia de las exigencias por encontrar el mejor programa de formación para las nuevas generaciones, con el objetivo de que sean capaces de responder al sistema social en el que vivimos. Marchesi (2007, p.13) expone que «el acceso a la información y al conocimiento, los 
cambios de la familia y de los propios alumnos, las modificaciones en el mercado laboral, los valores sociales emergentes..., son algunas características de la sociedad del siglo XXI que afectan sin duda, al ejercicio de la profesión docente».

De ahí que no hallemos hasta la fecha ningún instrumento validado que soporte las cuatro competencias docentes más utilizadas en la literatura científica española.

A partir de ahí se ve la necesidad de aportar y enriquecer esta agrupación como se ve en la Figura 1 por una competencias demandadas en la sociedad de hoy como es la necesidad de desarrollar su potencial creativo (Boada-Grau Boada-Grau, Sánchez-García, Prizmic-Kuzmica, A., y Vigil-Colet, 2014) y así poder adaptarse a los cambios continuos que sufre en su tarea diaria (Díaz-Carrera, 2010).

Toda institución que comprenda la innovación, como la puesta en práctica de ideas creativas, es clave para adaptarse a su ambiente y por

\section{LA ESTRUCTURA MENTAL DEL LIDER CREATIVO O MAESTRO DEL CAMBIO}

\section{Motivador y Moderador, conecta con su poder personal, estimuländolo en otros}

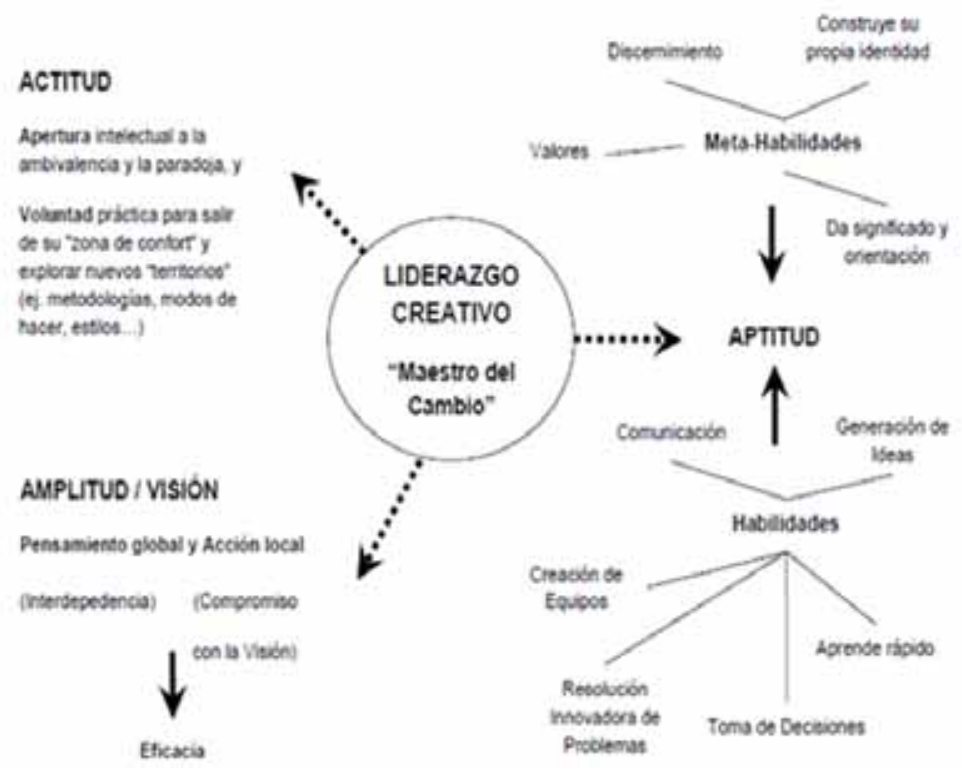

Figura 1. Estructura mental del líder creativo Nota Fuente: Díaz-Carrera, C. (2010, p. 246). tanto, será exitosa (Babicka, Dudek, Makiewicz, y Perzycka, 2010; Barroso, 2010 y 2011). Según Clegg y Birch (2001), sin creatividad se tomarán las mismas soluciones de siempre impidiendo la adaptación al medio. El docente, ha de tener una actitud tanto de apertura intelectual como voluntad para descubrir y aplicar nuevas metodologías y estilos de enseñanza, una amplitud/visión de miras que le hace comprometerse en conseguir el objetivo marcado y una aptitud en la que movilice la toma de decisiones, resolución de conflictos, para llegar a desarrollar meta-habilidades que le permita construir su identidad, adquirir valores. En este sentido, Romero, Zagalaz, Romero y Martínez (2011), apuntan competencias que permitan detectar y resolver problemas y apreciando y sabiendo atender a la diversidad comprometiéndose en potenciar el rendimiento académico. Hay que desarrollar sus habilidades comunicativas, trabajo en equipo y saber escuchar y respetar las opiniones de los otros, entendiéndose y trabajando conjuntamente (Pazo y Tejada, 2012)

Además, se demanda por parte de los estudiantes poder establecer una comunicación docente-discente de manera distinta a lo que se está realizando hasta ahora a través de una relación recíproca o lo que Not (1987), lo denomina el estatuto del co-sujeto. Define la educación como «constituyente» entendida como la formación o la transformación del profesor, el estudiante y el contexto. Viene a decir que en el proceso enseñanza-aprendizaje, la relación que se establece entre el profesor y el estudiante, no es simétrica ni paritaria sino que cada uno desde su posición, se condicionan mutuamente llevando a cabo actividades correlativas propias de la enseñanza y del aprendizaje asumiendo que cada uno es el «co-sujeto» del otro.

El alumno es protagonista de su propio aprendizaje y el profesor de su propia enseñanza. Pero además, aunque entre ambos exista un nivel de partida diferente, el profesor cuando enseña, aprende y el alumno cuando aprende es capaz de enseñar, construyendo una relación dialéctica entre ambos influyendo cada uno en el crecimiento personal del otro y por consiguiente en el de la sociedad.

Es necesario exponer al respecto que tanto en la propuesta de Hernández y Velázquez (2010, p. 74), como en las demás investigacio- 
nes, no se ha utilizado un instrumento validado. Es decir todavía estamos a la espera de hallar una herramienta capaz de identificar y confirmar cuales son las dimensiones que dibujan las competencias docentes del profesor de educación física. Somos conscientes de que la enseñanza es una empresa compleja y cambiante que no por ello vamos a dejar de analizar y en un futuro no muy lejano poder sistematizar.

\section{Futuras líneas de investigación}

Este trabajo permite tomar conciencia de la necesidad de unificar y acercar posiciones en cuanto a delimitar el concepto de perfil docente y saber qué competencias le definen como tal. En este intento de llegar a una aproximación de poder agrupar las competencias que todo docente ha de poner en práctica para dar respuesta a los cambios que sufre la sociedad, y definir una identidad docente acorde a los nuevos tiempos, es una realidad que a los docentes del siglo XXI se les demanda.

En primer lugar en cuanto a la relación y comunicación que ha de establecer con el estudiante y con el grupo clase, consiguiendo una relación dialéctica entre ambos. También se le demanda una capacidad de adaptación desarrollando un liderazgo creativo en sus clases que le permita realizar sus funciones de manera diferente con el objetivo de llevar a cabo con éxito todo el proceso enseñanza-aprendizaje.

Por tanto, es necesario seguir investigando en cómo diseñar programas y aplicaciones pedagógicas para mejorar la formación de formadores, tanto inicial como en la formación permanente tomando como referencias los modelos competenciales, para poder dar respuesta a los cambios educativos en el Siglo XXI.

Es clave elaborar programas de mejora de competencias docentes a través de la figura del «mentoring» aplicando cuestionarios validados antes y después del programa de intervención, constatando los resultados finales del profesor con la valoración realizada por los alumnos en cualquier área de conocimiento.

\section{Referencias}

Álvarez, G (2015). La cualificación de maestro en Europa: aportaciones a partir del análisis de las influencias supranacionales y los modelos europeos. Tendencias pedagógicas, 25, 9-34.

Babicka, A., Dudek, P., Makiewicz, M., y Perzycka, E. (2010). Competencia creativa del profesor. Revista electrónica interuniversitaria de formación del profesorado, 13(1), 51-61. Recuperado de http://www.aufop.com/aufop/uploaded_files/articulos/ 1268615940.pdf

Bandura, A. (1987). Pensamiento y acción. Fundamentos Sociales. Barcelona: Martínez Roca.

Barroso, F. (2010). Factores a considerar para desarrollar la creatividad en las empresas. Un estudio en 70 empresas de la ciudad de Mérida, Yucatán. XV Congreso Internacional de Contaduría, Administración e Informática (p. 42). México: Universidad Nacional Autónoma de México. Recuperado de http:// congreso.investiga.fca.unam.mx/docs/xv/resumen

Barroso, F. (2011). ¿Son aplicables los conceptos de la creatividad usados en la Administración de Empresas, a la gestión del personal de centros educativos? Investigación universitaria multidisciplinaria, 10(10), 37-46.

Boada-Grau, J., Sánchez-García, J., Prizmic-Kuzmica, A., y Vigil-Colet, A. (2014). Adaptación española de la escala de Potencial Creativo y de la Creatividad Practicada (CPPC-17) en el trabajo y en la organización. Psicothema, 26(1), 55-62.

Bourgonje, P., y Tromp, R. (2011). Educadores de Calidad: Estudio internacional sobre las competencias y estándares para docentes. UNESCO: Internacional de la educación. Oxfam Novib.

Bridges, E. M. (1986). The incompetent Teacher. The callenge and the response. Filadelfia: Falmer Press.

Carreiro da Costa, F. (2006). La Enseñanza de la Educación Física ante la Implantación del Espacio Europeo de Educación Superior. Educación Física y Deporte, 25(2), 29-43.

Clegg, B., y Birch, P. (2001). Creatividad al instante. México: Granica.

Comisión Europea (2013). Supporting teacher competence development for better learning outcomes Disponible en: http://ec.europa.eu/education/policy/school/doc teachercomp en.pdf

Consejería de Educación de la Comunidad de Madrid. (2001). Informe de la valoración de la labor docente. Madrid: Autor.

Consejería de Educación y Ciencia. (2007). El educador en la sociedad del siglo XXI. Recuperado de http://www.jccm.es/educacion/consejo_e/informes/ informe_educador_

Consellería d’Educació. Generalitat Valenciana. (2010). Propuesta para la evaluación del profesorado en los niveles no universitarios. Valencia: Generalitat Valenciana.

Danielson, CH. (2011). Competencias docentes: desarrollo, apoyo y evolución. Marco para la Enseñanza. PREAL Programa de Promoción de la Reforma Educativa en América Latina y el Caribe. Recuperado de http://www.gse.upenn.edu/pdf/ Competencias\%20docentes.pd
Del Valle, S., De la Vega, R., y Rodríguez, M. (2015). Percepción de las competencias profesionales del docente de educación física en primaria y secundaria. Revista internacional de medicina y ciencias de la actividad física y el deporte, 15(59), 507-526.

Departamento de Educación del Estado de Ohio. (2015). Estándares de desempeño docente. Recuperado de http://education.ohio.gov/Topics/Teaching/EducatorEvaluation-System/Ohio-s-Teacher-Evaluation-System/Teacher-PerformanceRatings

Departamento de Educación, Cultura y Deporte. Gobierno de Aragón (2013). Guía para la evaluación de la función docente Dimensiones/Subdimensiones/Indicadores. Recuperado de

http://www.educaragon.org/Files/Files/UserFiles/File/Inspeccion\%2013-14/ Gua\%20DOCENS\%20general.pdf

Department of Education. Western Australia. (2009). Level 3 Class-room Teacher. Recuperado de http://www.det.wa.edu.au/detems/teachingwa/

Díaz-Carrera, C. (2010). El líder como generador de sentido. Revista internacional de pensamiento político, 5, 239-248.

Díaz, F., Blázquez, P. J., Casse, J. L., Díaz, F., Escudero, J., Martínez, A. M., (...)., y Sánchez, G (2010). Modelo para autoevaluar la práctica docente. Madrid: Wolters Kluwer España. S. A.

Dirección General de Calidad, Innovación y Formación del Profesorado (2010). Modelo de competencias profesionales del profesorado. Castilla y León: Autor.

doi: http://dx.doi.org/10.15366/rimcafd2015.59.007

Ferrández-Berrueco, R., y Sánchez-Tarazaga, L. (2014). Competencias docentes en secundaria. Análisis de perfiles de profesorado. RELIEVE, 20(1), doi: 10.7203/relieve.20.1.3786

Gairín, J. (2011). Formación de profesores basada en competencias. Bordón, 63(1), 93108.

Galvis, R., Fernández, B., y Valdivieso, M. (2007). Construcción de perfiles por competencias bajo el enfoque del marco lógico. Taller presentado en el Congreso Internacional de Calidad e Innovación en Educación Superior.

Hernández, J. L., y Velázquez, R. (Coord.) (2010). La educación física a estudio. El profesorado, el alumnado y los procesos de enseñanza. Barcelona: Graó

Jofré, G, y Gairín, J. (2010). Competencias profesionales de los docentes de enseñanza media de Chile. Un análisis desde las percepciones de los implicados. Barcelona: UAB-Departamento de Pedagogía Aplicada.

Junta de Andalucía. Consejería de Educación. (2011). Estándares de la práctica profesional docente en Andalucía. Sevilla: Autor.

Lavega, P. (2008). Educación Física y mercado laboral. Competencias profesionales. Calle libre. Cultura, Ciencia y Deporte, 8(3), 123-131.

Marchesi, A. (2007). Sobre el bienestar de los docentes. Competencias, emociones y valores. Madrid: Alianza Editorial.

Marcelo, C. (2002). Los profesores como trabajadores del conocimiento. Certidumbres y desafíos para una formación a lo largo de la vida. Educar, 30, 27-56, doi: http:// dx.doi.org/10.5565/rev/educar.312

Martínez, M. (2016). La formación inicial de los maestros: una responsabilidad compartida. Bordón, 68(2), 9-16.

Ministerio de Educación de Chile. (2003). Marco para la Buena Enseñanza. Recuperado de http://www.rmm.cl/usuarios/equiposite/doc/200312031457060.mbe.pdf

Ministerio de Educación de Ecuador. (2011). Estándares de desempeño profesional docente. Propuesta para la discusión ciudadana. Quito, Ecuador: EOI.

Ministerio de Educación de Perú. (2012). Marco de Buen Desempeño Docente. Perú: Autor.

Ministerio de Educación de República Dominicana. (2012). Modelo de evaluación del desempeño docente basado en competencias de la República dominica. Santo Domingo: IDEICE. Instituto dominicano de evaluación e investigación de la calidad educativa.

Not, L. (1987). El estatuto del co-sujeto en las situaciones pedagógicas. Educar, 11, 7993.

OECD (2016). Education at a Glance 2016: OECD Indicators. OECD Publishing. Recuperado de http://dx.doi.org/10.187/eag-2016-en

Pazo, C. I., y Tejada J. (2012). Las competencias profesionales en Educación Física Retos. Nuevas tendencias en Educación Física, Deporte y Recreación. 22, 5-8

Perrenoud, P. (2004). Diez nuevas competencias para enseñar. Barcelona: Graó.

Red de Formación del Profesorado de la Xunta de Galicia (2014). Las competencias profesionales docentes. Recuperado de http://www.edu.xunta.es/centros/cafi/ aulavirtual 2 /file.php/ 1 / competencias_profesionais / competencias profesionales docentes.pdf

Reforma Educativa en el Aulay $\mathrm{F}$ 